\title{
Linx
}

Revue des linguistes de l'université Paris X Nanterre

$11 \mid 1999$

Typologie des langues, universaux linguistiques

\section{Le marquage du bénéficiaire/expérient en grec et en albanais : peut-on généraliser la notion de relation dative dans la comparaison typologique?}

\section{Georges Drettas}

\section{OpenEdition}

\section{Journals}

Édition électronique

URL : http://journals.openedition.org/linx/951

DOI : $10.4000 /$ linx.951

ISSN : 2118-9692

Éditeur

Presses universitaires de Paris Nanterre

\section{Édition imprimée}

Date de publication : 1 décembre 1999

Pagination : 235-254

ISSN : 0246-8743

\section{Référence électronique}

Georges Drettas, « Le marquage du bénéficiaire/expérient en grec et en albanais : peut-on généraliser la notion de relation dative dans la comparaison typologique ? », Linx [En ligne], 11 | 1999, mis en ligne le 03 juillet 2012, consulté le 19 avril 2019. URL : http://journals.openedition.org/linx/951 ; DOI :

$10.4000 /$ linx.951

Ce document a été généré automatiquement le 19 avril 2019.

Département de Sciences du langage, Université Paris Ouest 


\title{
Le marquage du bénéficiaire/expérient en grec et en albanais : peut-on généraliser la notion de relation dative dans la comparaison typologique?
}

\author{
Georges Drettas
}

\section{Introduction ${ }^{1}$}

1 Dans le moment des origines, alors que le structuralisme post-saussurien se constituait, la région des Balkans, avec ses cultures et ses langues, a servi d'illustration typique à la notion d'aire de convergence, que le Prince Troubetzkoy proposait dans la phase où il élaborait les fondements de la phonologie (v. G. Drettas, 1986). L'inventaire des systèmes l'amenait à mettre en lumière aussi bien les similarités structurelles que les différences. Or, les similarités systémiques suscitaient l'intérêt dans le cadre d'interrogations typologiques dont certaines n'étaient pas nouvelles, à ceci près qu'elles s'étaient longtemps cantonnées à des questions de morphologie.

2 Le fameux ouvrage de K. Sandfeld, La Linguistique balkanique, publié en 1930, marque un moment fondateur où l'on voit se constituer l'étude des langues de la région en une sorte de modèle méthodologique. On constate, toutefois, que la phonologie proprement dite n'a pas investi le domaine. L'intérêt se concentre sur deux ensembles de faits linguistiques : a) La morphologie et la syntaxe ; b) Le lexique, entendu au sens large. Précisons que les questions lexicales (emprunts, phraséologie, etc.) restent aujourd'hui encore le thème dominant des études balkanologiques.

Dans cette approche, l'attention des «balkanologues» s'est focalisée sur les traits communs à l'ensemble des langues concernées. On établit l'inventaire des fameux balkanismes primaires, renvoyant à une trajectoire diachronique que l'on pourrait résumer 
au moyen de la proposition emblématique : «la morpho-syntaxe des langues balkaniques manifeste une évolution du synthétisme à l'analytisme ».

4 L'analytisme s'illustre, par exemple, dans la réduction du nombre des formes casuelles (grec moderne, roumain) ou dans leur disparition totale (bulgaro-macédonien, aroumain). Le développement de certaines formes verbales à auxiliaire (parfait, futur, etc.) est également conçu comme relevant de l'analytisme.

5 Curieusement, on ne comparait pas systématiquement les langues qui étaient à l'évidence le plus «similaires ». En particulier, le grec et l'albanais, qui auraient dû, logiquement, faire l'objet d'un examen approfondi, ont été isolés pour des raisons purement politiques, et cela jusqu'à la fin du régime de Enver Hoxha. En termes imagés, on pourrait parler ici du refoulement d'une évidence que la perspective diachronique elle-même renforçait.

6 Si l'on a parlé très tôt d'une tendance à la perte du datif, hérité du système indoeuropéen, manifestée en grec ( $\mathrm{du}$ grec post-classique au grec moderne) et en bulgare (disparition des cas du vieux-bulgare), la comparaison nécessaire avec les faits de l'albanais a été longtemps bloquée pour les raisons idéologiques que je viens de mentionner. Il n'est pas sans intérêt de remarquer, du reste, que la proximité typologique du grec et de l'albanais, si apparente dans la synchronie, renvoyait aux affinités diachroniques que les lignes officielles avaient pour fonction de masquer, aussi bien en Albanie et au Kosovo qu'en Grèce.

7 Ces aléas historiques ne nous retiendront pas davantage et nous allons porter notre attention sur le point de méthode qu'implique la question évoquée. En effet, même avec des étiquettes relativement imprécises, la démarche comparative aurait dû commencer par l'examen des systèmes casuels grecs et albanais afin d'inclure, ultérieurement, les observations ordonnées dans un ensemble plus large qui dépasserait les limites de langues génétiquement apparentées.

8 Je me propose de reprendre ici cet examen, de façon certes partielle, et cela dans la perspective suivante: La typologie contemporaine peut débloquer la balkanologie classique, en dépit de son hétérogénéité théorique. L'effort pour surmonter le "passif idéologique » avait déjà commencé dans la linguistique soviétique des années 70, mais les meilleures tentatives (Civjan, Lopašov, Maslov, etc.) s'essoufflaient assez vite par défaut factuel.

9 L'ensemble albanais (R.P. d'Albanie et Kosovo) disposait d'excellents linguistes, mais ceux-ci ne s'intéressaient plus au grec, pour les raisons déjà mentionnées. Il faut attendre 1986, lorsque le Prof. Shaban Demiraj publie sa Grammaire historique de la langue albanaise. Entre temps, le contexte historique avait été négatif pour l'étude diachronique de l'albanais, qui est une langue portée à l'écrit tardivement, mais aussi pour le grec. Ces deux langues voisines étaient en effet présentes dans l'espace balkanique avant l'installation des groupes roman et slave.

Quant aux rapprochements avec d'autres familles, il semble normal de les effectuer. Pour des raisons de cohérence aréale dans la longue durée, on pense en tout premier lieu aux langues du Caucase du Sud (langues kartvèles) voire même, pour certains faits, aux langues du Caucase du Nord-Ouest (Adyghe, Abxaz, etc.).

11 Une comparaison, même rapide, des phénomènes de marquage actanciel fait apparaître au sein de l'objectalité le caractère fondamental, voire premier, de l'élément appelé « complément d'object indirect » ou « complément d'attribution » dans nos grammaires traditionnelles. Afin de faciliter l'examen des données, il semble nécessaire de préciser les 
étiquettes employées. Par "datif» nous entendons la marque casuelle définie dans la tradition de langues comme le grec ancien, le latin, le vieux-slave ou l'allemand. Une des fonctions centrales de ce cas est de marquer un nom (pronom, relatif, SN, etc.) correspondant au rôle sémantique de Bénéficiaire/Expérient. La « relation dative » met en jeu un participant central correspondant formellement à un Bénéficiaire/Expérient ou «attributaire " dans les formules de possession du type «cette table est à moi ». La relation n'implique pas l'existence d'un système casuel dans les nominaux. Cela dit, un tel système est souvent résiduel, limité au paradigme de pronoms atones ou d'indices personnels intra-verbaux, comme on peut en observer dans les langues romanes, les langues indo-européennes des Balkans (grec, bulgare, albanais) ou les langues du Caucase méridional (géorgien, laze, svane). Notre perspective vise à opérer en typologie avec une notion de dativité qui soit similaire, dans la comparaison des langues, avec l'accusativité ou l'ergativité couramment admises.

\section{Actance, verbe et cas en albanais et en grec}

Comme dans les langues balkaniques voisines (serbe, bulgare, aroumain), l'albanais et le grec moderne sont des langues à opposition verbo-nominale tranchée. Dans ces langues, le Syntagme Verbal est nécessaire et suffisant pour constituer un énoncé bien formé dans des conditions pragmatiques données. Compte tenu de ce fait, on peut considérer que les Syntagmes Nominaux sont, formellement, apposés au SV qui les régit. Ce dernier peut être mono-, bi- ou triactanciel. Il porte obligatoirement la marque d'au moins un actant, lequel est considéré traditionnellement comme le «sujet». Cet élément peut correspondre aux rôles sémantiques d'Agent, de Patient ou de Bénéficiaire/Expérient. La marque subjectale ordonne le classement des paradigmes verbaux dans les grammaires, soit la $1^{\text {ère }}, 2^{2 \text { ème }}, 3^{\text {ème }}$ personne, etc.

Le système verbal repose sur une opposition aspectuelle fondamentale perfectif/ imperfectif. Le perfectif est représenté par la forme appelée aoriste et par le parfait périphrastique, là où il existe. L'imperfectif, lui, est représenté par deux formes, le présent et l'imparfait. Ces étiquettes traditionnelles ont un sens purement morphologique. Enfin, les particules modales (do-, do-të, të-, en albanais; 䢙 $a-$-, na-, as-, en grec) se préfixent aux formes précédentes pour réaliser des "aspects-modes " (futur, optatif, inférentiel, etc.).

Enfin, il existe une diathèse moyenne, réalisée le plus souvent par un couple Verbe "actif»/Verbe "moyen" en - $m$. Il s'agit en fait de la 1p. sing. prés. qui, par convention, sert à identifier cet ensemble. Je rappellerai que le moyen ou médio-passif est connu dès les états les plus anciens du grec, et qu'il existe aussi en arménien sous une forme très similaire à celle du grec ou de l'albanais. Exemples :

a) albanais : laj « je lave qqch » / lahem « je me lave, on me lave »; mendoj « je pense, j'ai l'opinion que » / mendohem « je pense, je réfléchis».

L'aoriste moyen se forme par la préfixation de $u$ - à l'aoriste "actif": lava «j'ai

lavé » / u.lava « je me suis lavé ».

b) grec : 'plino « je lave » / 'plinome « je me lave » / 'plitika « je me suis lavé ».

En grec, comme en albanais, nombre de verbes moyens sont isolés. Un exemple : 'skeftome «je pense, je réfléchis ». 
En albanais, si le nom est non défini, ou « indéterminé » selon la terminologie courante, $\mathrm{i}$ présente deux formes correspondant à un cas direct et un cas oblique. Notons que dans les grammaires albanaises, la forme oblique, qui est identique au nominatif de la forme définie, est présentée comme correspondant aux cas génitif, datif, accusatif. Exemples :

\begin{tabular}{|c|c|c|c|c|c|}
\hline \multicolumn{2}{|l|}{ a) masculin } & \multicolumn{2}{|l|}{ b) féminin : } & \multicolumn{2}{|l|}{ c) pluriel : } \\
\hline cas direct: & cas oblique & cas direct & cas oblique & cas direct & cas oblique \\
\hline lis « chêne " & lisi & fushë « plaine » & fusha & lisa " chênes " & lisave \\
\hline burrë « garçon» & burri & motër « soeur » & motra & & \\
\hline ftua « coing » & ftoj & & & & \\
\hline zë « voix» & zëri & & & & \\
\hline
\end{tabular}


21 Je laisse de côté la forme génitivo-adjectivale en -sh, qui n'est pas pertinente pour notre propos.

Le tableau suivant présente de façon synthétique la morphologie nominale définie. Je précise que les connecteurs fonctionnent soit comme des articles préposés indiquant la définitude du SN (par exemple, $i$ biri «le fils»), soit comme des marqueurs de détermination entre deux SN, comme l'illustre le tableau des exemples empruntés à la description de Hans Jürgen Sasse (1991).

Noms définis :

\begin{tabular}{|c|c|c|c|c|c|c|}
\hline & & connecteurs & \multicolumn{4}{|c|}{ cas } \\
\hline & & & nominatif & accusatif & datif & génitif \\
\hline \multirow{2}{*}{ Sing. } & masculin & i & $-i,-u$ & -n(ë) & \multicolumn{2}{|c|}{-it, -ut } \\
\hline & féminin & $e, t e ̈$ & $-\mathbf{a},-\mathbf{j a}$ & $-($ ) në & \multicolumn{2}{|c|}{$-($ ) së } \\
\hline \multicolumn{2}{|c|}{ pluriel } & të, (së) & \multicolumn{2}{|c|}{-()$t(\ddot{e})$} & \multicolumn{2}{|c|}{-ve, -ve(t) } \\
\hline
\end{tabular}

Exemples:

\begin{tabular}{|c|c|c|c|c|c|}
\hline \multirow{4}{*}{$\begin{array}{l}\text { Nmasc burëë "garçon " } \\
\text { N fém, fushë "plaine " }\end{array}$} & & nominatif & accusatif & datif & génitif \\
\hline & \multirow{2}{*}{ singulier } & burr.i & burr.in & burr.it & i/e-burr.it \\
\hline & & fush.a & fushë.n & fushë.s & i/e-fushë.s \\
\hline & pluriel & \multicolumn{2}{|c|}{ burra.t } & \multicolumn{2}{|c|}{ burra.ve } \\
\hline
\end{tabular}

Détermination nominale. Exemples :

\begin{tabular}{|c|c|c|c|}
\hline \multirow[t]{4}{*}{ masculin } & nomin. & sing. : & trapéz.i $\underline{\text { i-máo }}$ « la grande table »(litt. " la table la grande ») \\
\hline & & plur. : & $\begin{array}{l}\text { trapézeta tə-mbəŏen « les grandes tables } * \text { (litt. « les tables les } \\
\text { grandes } *)\end{array}$ \\
\hline & "génitif" & $:$ & sí.u i-djánit « l’oil de l'enfant »(litt. « l'oeil le de l'enfant ") \\
\hline & acc. & : & sí.nə ta-djá́it * l'œil de l'enfant »(litt. « l'oeil le de l'enfant ") \\
\hline & & $:$ & sakáj.nə tà-rí « la veste la nouvelle* \\
\hline féminin & $\begin{array}{l}\text { sing.: } \\
\text { plur.: }\end{array}$ & & 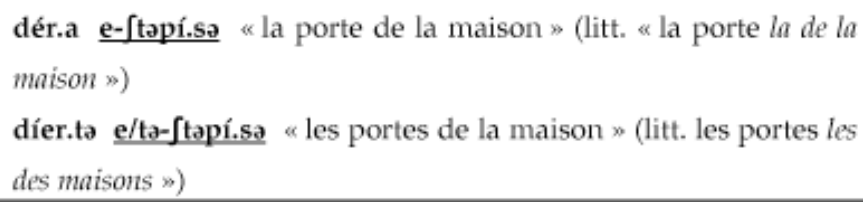 \\
\hline
\end{tabular}

Considérant que le SN Objet d'une construction transitive est au cas accusatif, comme en grec (cf. infra), alors que le $\mathrm{SNO}_{2}$ (ou troisième actant) est au datif/génitif, on dira que la structure actancielle de base est accusative. Il faut bien sûr nuancer sensiblement cette proposition, car l'esquisse morphologique nous amène à constater que l'opposition étiquetée Nominatif/Accusatif est formellement limitée au singulier pour le SN défini.

On remarque l'identité formelle du génitif et du datif, la relation de détermination intranominale étant assurée par les connecteurs $i-/ e-, t \ddot{e}-$, së-.

Il convient de souligner le fait que le système à trois marques, nominatif / accusatif / datif, du singulier défini correspond au système à deux marques du singulier non défini et du pluriel défini ou non défini. Là le datif bien stable s'oppose formellement à ce qu'on pourrait considérer comme une forme absolutive. La situation formelle du $\mathrm{N}$ déf. plur. est 
cruciale en ce qu'elle rappelle évidemment le comportement de la classe des $\mathrm{N}$ neutres du grec, dans les dialectes qui utilisent le génitif/datif comme marque actancielle, ou encore celle des dialectes lazes qui n'ont pas la marque ergative $-k$ pour spécifier l'Agent d'une construction transitive.

Avant d'examiner les données du grec, il convient de revenir au SV de l'albanais, puisqu'il constitue le pivot du système d'accord qui permet de fonder la caractérisation typologique d'une construction (accusative, ergative, inverse, etc.). Les indices objectaux:

En situation d'anaphore, le SV bi- ou tri-actanciel se voit affixer les indices objectaux suivants, qui représentent d'anciens pronoms atones:

\begin{tabular}{|l|l||l|l|l|l|}
\hline & & \multicolumn{2}{|c|}{ singulier } & \multicolumn{2}{c|}{ pluriel } \\
\hline & & objet $_{1}$ & objet $_{2}$ & objet $_{1}$ & objet $_{2}$ \\
\hline & & më- & \multicolumn{2}{|c|}{ na-, ne- } \\
\hline & $2 p$. & të & \multicolumn{2}{|c|}{ ju- } \\
\hline $2 p$. & e- & i- & i- & u- \\
\hline
\end{tabular}

Les normes de la langue actuelle nous disent que la présence d'un SN défini dans une construction transitive entraîne l'accord objectal obligatoire. Le terme, emprunté à T. Givón, désigne le fait d'utiliser la formule actancielle maximale du SV, dans un contexte où les besoins anaphoriques ne le justifieraient pas. Afin d'illustrer ce phénomène, j'emprunterai un exemple simple à la Grammaire historique de Sh. Demiraj (p. 569) :

\begin{tabular}{|l|l|l|}
\hline 1 & 2 & 3 \\
\hline e-bleva & librin & pardje \\
\hline $\begin{array}{l}\text { 1/ obj. 3p. sing. + V « acheter » 1p. sing. aor. 2/ N « livre » masc. sing. acc. 3/ temp. « avant- } \\
\text { hier ». } \\
\text { litt. : « je l'ai acheté le livre avant-hier ». }\end{array}$ \\
\hline
\end{tabular}

Des travaux récents ont montré que le caractère absolu des normes d'accord doit être relativisé en fonction des paramètres énonciatifs. Nous retiendrons ici que, de ce point de vue, le troisième actant (ou $\mathrm{O}_{2}$ ) est prioritaire. En d'autres termes, le datif passe avant l'accusatif, dans la mesure où l'accord objectal est obligatoire avec l'actant correspondant au Bénéficiaire. La contrainte d'accord est hiérarchisée en faveur du " datif », dans ce qui ressemble fort à la conjugaison objective de certaines langues (hongrois, etc.).

Pour en revenir à l'aspect purement formel des faits, nous observons le clivage entre la 3 p. sing./plur. et les 1 et 2 p. sing. et plur., qui ont la même forme pour l'objet 1 (accusatif) et l'objet ${ }_{2}$ (datif). 
Dans une partie du système, le domaine objectal réunit dans une même marque le Patient et le Bénéficiaire. Avec une répartition légèrement différente, l'ensemble grec présente des phénomènes similaires.

\section{B. Marques casuelles en grec}

Noms définis. Exemples (pontique) :

\begin{tabular}{|c|c|c|c|c|c|c|}
\hline & \multicolumn{3}{|c|}{ singulier } & \multicolumn{3}{|c|}{ pluriel } \\
\hline & nominatif & accusatif & génitif & nominatif & accusatif & génitif \\
\hline mescalin & o'-arசop.o.n & ton-'arep.o.n & t- a'r $\theta$ op & i- a'r $\theta$ op & t- a'r0op.s & t- $\operatorname{ar} \theta 0$ 'p.ion \\
\hline féminin & i- $\gamma$ a'r.i & tin- $\gamma$ a'r.in & ti- $\gamma a^{\prime} r$ is & i- $\gamma$ a'ri.ð.es & ta- $\gamma$ a'ri. $\partial$.as & ti- $\gamma$ ari'. $\delta$.ion \\
\hline neutre & \multicolumn{2}{|c|}{ to- xo'raf } & ti- xora'f.i & \multicolumn{2}{|c|}{ ta- xo'raf,æ } & ti- xora'f.ion \\
\hline
\end{tabular}

Le dialecte pontique est pris ici comme exemple, car il permet d'illustrer de façon simple et régulière les principes morphologiques communs au diasystème. Ce dernier présente la répartition suivante des marques actancielles :

- Groupe A (Grèce du Sud, îles ioniennes, Crète, arc insulaire oriental), correspondant à la base normative de la dhimotiki écrite, à l'exception de l'ensemble dodécanésien et chypriote :

1) Classe des masculins/féminins: Système à trois cas, nominatif-accusatif-génitif. Le nominatif marque l'actant unique ou le premier actant, correspondant à l'accord subjectal. L'accusatif marque le second actant de la construction transitive et le génitif opère comme marque de détermination nominale, soit comme marque du troisième actant ou, plus simplement, du datif, à l'instar de ce que nous avons vu en albanais.

2) Classe des neutres : Système à deux cas, nominatif(accusatif)-génitif(datif). Cette formule rappelle la situation des $\mathrm{N}$ non définis ou des $\mathrm{N}$ pluriels en albanais.

- Groupe B (dialectes septentrionaux : Roumélie, Thessalie, Macédoine, Thrace, Limnos, Mitylène, Sporades, Samothrace, Asie Mineure [Pont, Farasa, Cappadoce], Crimée).

Ce qui caractérise cet ensemble de dialectes, par ailleurs très différents entre eux, c'est le fait que l'accusatif assume le marquage de la relation dative, le génitif fonctionnant exclusivement dans la détermination nominale. La classe neutre donc est en dehors du système casuel, du point de vu actanciel.

Les indices objectaux (dhimotiki):

Le SV transitif peut se voir affixer les indices objectaux suivants :

\begin{tabular}{|c|c|c|c|c|c|}
\hline & & \multicolumn{2}{|c|}{ singulier } & \multicolumn{2}{|c|}{ pluriel } \\
\hline & & objet $_{1}$ & Objet $_{2}$ & objet $_{1}$ & Objet $_{2}$ \\
\hline $1 p$. & & me- & mu- & \multicolumn{2}{|c|}{ mas- } \\
\hline $2 p$. & & se- & su- & \multicolumn{2}{|c|}{ sas- } \\
\hline $3 p$. & masc. & ton- & tu- & \multicolumn{2}{|c|}{ tus- } \\
\hline
\end{tabular}




\begin{tabular}{|l|l|l|l|l|l|l|}
\hline & & fém. & tin- & tis- & \multicolumn{2}{|l|}{} \\
\hline & & neut. & to- & tu- & ta- & tus- \\
\hline
\end{tabular}
exemples :

Remarques sur la séquence affixale :

De ce point de vue, trois possibilités se partagent l'ensemble dialectal :

a) Les indices objectaux sont proclitiques, comme en albanais. Le SV maximal type correspond à la formule : \| particule modale/négation $+\mathrm{O}_{2}+\mathrm{O}_{1}+\mathrm{V}(\mathrm{s}) \|$. Cet ordre est aussi bien celui des dialectes méridionaux (Péloponnèse, etc.) que septentrionaux (Macédoine, Thrace, etc.) et c'est celui de la dhimotiki actuelle.

b) L'ordre des indices est mixte. Dans les énoncés assertifs simples, les indices objectaux sont enclitiques, suffixés à la base. Ils sont proclitiques, si le SV est préfixé d'une particule modale et/ou négative. Cette formule est présente dans l'ensemble insulaire oriental (rhodien, chypriote, etc.). La séquence était attestée dans les dialectes de Cappadoce (Aksu, Farasa, etc.).

- Dans les cas a) et b), les indices sont toujours enclitiques dans l'énoncé injonctif, de même qu'en albanais.

c) L'ordre des indices est: enclitique pour tous les énoncés. C'est la séquence du grec oriental, pontique et gréco-criméen. Rappelons que c'était celui du grec de la Septante. La formule du SV maximal est donc: $\|$ part. mod. (+ nég.vb.) $+\mathrm{V}(\mathrm{s})+\mathrm{O}_{2}+\mathrm{O}_{1}(+$ part. foc.) $\|$.

\section{L'accord objectal}

L'accord objectal est certes moins obligatoire en grec qu'en albanais. Son caractère plus ou moins contraignant dépend de la composante énonciative (pragmatique). Par exemple, un SN défini objet (ou objet $_{2}$ ) implique presque toujours l'accord objectal, s'il est thématisé. En pontique en revanche, la thématisation forte d'un SNobjet exclut l'accord objectal (v. Drettas, 1997). Il convient d'apporter deux précisions avant de présenter quelques

1) Lorsque la marque indicielle est identique pour le Patient et le Bénéficiaire (comme au pluriel, en grec, ou aux 1 et 2p. sing., en albanais), il n'y a pas d'ambiguïté référentielle, en raison de la séquence. Comparons, par exemple, les deux énoncés qui suivent :

$\begin{array}{lll}1 & 2 & 3\end{array}$

a. ton- pa'tera-mas ton-'skotosan sto-vu'no

$1 /$ art. $+\mathrm{N}$ "père " masc. sing, acc. + poss. 1 p. plur. = " notre père ».

$2 /$ obj. $_{1} 3$ p. masc. plur. $+V$ « tuer $" 3 p$. plur. aor. $=$ «ils l'ont tué, on l'a tué ".

3 / loc.art. $+\mathrm{N}$ « montagne " = « à la montagne ".

"Notre père, on l'a tué dans le maquis".

a'. ton-pa'tera-mas | mas-ton-'skotosan sto-vu'no
notre père on nous l'a tué dans le maquis

Avec l'indice objet de 1p. plur. -mas, la séquence \#mas- 'skotosan \# ne peut signifier que « on nous a tués ». On peut presque toujours indiquer la personne au bénéfice ou au 
détriment de qui une action a été accomplie. Les langues romanes n'ignorent pas cette formulation du Bénéficiaire. Mais on pense aussi aux langues kartvèles qui font un large usage de ce qu'on appelle les voyelles de version pour orienter le SV transitif.

2) Le grec et l'albanais font un usage important de la construction inverse pour dénoter des processus d'affectation de l'un des actants, lequel est en position d'Expérient (Experiencer, dans la terminologie anglo-saxonne). Pour traduire, par exemple, l'énoncé «j'ai besoin d'un crayon », on peut avoir, en grec :

$$
123
$$

a. mu-xri'azete 'ena mo'livi

$1 /$ obj.2 1p. sing. + V moyen "nécessiter, avoir besoin » 3p. sing. prés.

$2 /$ numér. " un " neut. sing. $3 / \mathrm{N}$ « crayon " neut. sing.

\section{a'. xri'azome 'ena mo'livi}

\begin{tabular}{|l} 
e'mena mu-xri'azete 'ena mo'livi $=$ "j'ai besoin d'un crayon, moi ». \\
\hline Je précise que, dans ce cas, l'accord objectal est obligatoire. \\
\hline 'ena mo'livi to-xri'azome = « un crayon, j'en ai besoin “.
\end{tabular}

Exemples albanais :

1. Dhe $e^{1}$ dialit $^{2} i$-dha ${ }^{3}$ çupën ${ }^{4} e$-vet ${ }^{5}$ edhe $e^{6}$ e-bëri $i^{7}$ dhëndër ${ }^{8} 1 /$ coord. « et ». 2/ N « garçon » masc. sing. dat. déf. $3 /$ obj. 3p. sing. dat. $+\mathrm{V}$ « donner » 3p. sing. aor. $=$ « il lui donna ». 4/ art. $+\mathrm{N}$ « fille » fém. sing. acc. $5 /$ art. $+\mathrm{N}$ poss. $4 /+5 /=$ litt. « la fille la sienne » = «sa fille ». 6/ coord. « et ». 7/ obj. 3p. sing. acc. $+\mathrm{V}$ « faire » 3p. sing. aor.. 8/ $\mathrm{N}$ « gendre » masc. sing. acc. non dét. $7 /+8 /=$ « il le fit gendre (= il en fit son gendre) ».

«Et il donna sa fille au garçon et il en fit son gendre ».

2. \# ndjente ${ }^{1} s^{2}$ po-e-linte ${ }^{3}$ fuqia $^{4}, s^{5}$ po-i-afrohej $^{6}$ vdekja $^{7}$, dhe $^{8}$ nuk-donte ${ }^{9}$ të-jepej ${ }^{10}$. Poafronte ${ }^{11}$ koha $^{12} e$-draprit ${ }^{13}$ të-parë ${ }^{14}$ dhe ${ }^{15} e$-shtegtimit ${ }^{16}$ të-bagëtive ${ }^{17}$. Memoja ${ }^{18} u$-mendua ${ }^{19}$. E-zuri ${ }^{20}$ për-krahu ${ }^{21}$ komandantin $^{22}$ Rrapo $^{23} e^{24}$ i-tha ${ }^{25}$ : - Të-dëgjojmë Zarikun \#

$1 / \mathrm{V}$ « sentir, ressentir» 3p. sing. impft. 2/ part. mod. "que». 3/ part. asp. + obj. 3p. sing. + $\mathrm{V}$ « libérer, vider »3p. sing. impft. $4 / \mathrm{N}$ « force » dét. fém. sing. nomin. $5 /$ »que ». 6/ part. asp. + obj. 2p. sing. + V moyen « s'approcher » 3p. sing. impft. 7/ N « mort » dét. fém. sing. nomin. 8 / »et ». 9/ part. nég. + $\mathrm{V}$ « vouloir » 3p. sing. impft. 10/ part. mod. + V moyen « se donner ». 11/ part. asp. $+\mathrm{V}$ « approcher, rapprocher » 3p. sing. impft. 12/ N « temps » fém. sing. nomin. 13/ $\mathrm{N}$ « faucille » dét. sing. génit. 14/ $\mathrm{N}$ adj. « premier » sing. 15 / coord. « et ». 16/ N « cheminement, transhumance » dét. masc. sing. génit. 17/ N «bêtes, ovins et caprins " dét. plur. génit. 18/ $\mathrm{N}$ "Mehmet » dét. nomin. sing. 19/V moyen « penser, réfléchir» 3p. sing. aor. 20/ obj. + V « prendre, saisir» 3p. sing. aor. $21 /$ relat. "pour " $+N$ « bras, épaule » déf. masc. sing. acc. 22/ $N$ « commandant» dét. masc. sing. acc. 23/ $\mathrm{N}$ anthropon. « Rapo ». 24/ coord. »et ». 25/ obj. 3p. sing. dat. + V 
« dire » 3p. sing. aor. = « il lui a dit ». 26/ part. mod. + V « entendre, écouter » 1p. plur. prés. 27/ N anthropon. « Zarik» déf. masc. sing. acc.

«Il sentait que la force le quittait (litt. se vidait lui), que la mort se rapprochait de lui, et il ne voulait pas se laisser aller (litt. se donner). Le temps de la première faucille et de la transhumance des bêtes approchait déjà. Memo réfléchit. Il saisit par le bras le commandant Rapo et il lui dit : - Nous devons écouter Zarik ».

Cet exemple un peu long a le mérite d'illustrer plusieurs des faits formels que nous avons présentés plus haut. Emprunté au célèbre roman de Dritëro Agolli Komisari Memo, il propose également la norme du tosk écrit après 1944. On y repère plusieurs SV avec l'accord objectal, ainsi qu'un SV combinant un verbe moyen et un objet datif (6). Cette possibilité est attestée dans le lexique de la langue (cf. N. Gjini, 1971). Dans son travail consacré à la syntaxe des formes pronominales atones, R. Përnaska (1996) donne de nombreux exemples de telles constructions.

Il me semble que, dans ces formules très fréquentes aussi bien en albanais qu'en grec, nous avons bien deux actants. Autres exemples :

3. mua ${ }^{1}$ më-dhemb ${ }^{2}$ koka $^{3}$

$1 / \mathrm{N}$ pers. " moi " acc. $2 / \mathrm{obj}_{1} 1$ p. sing. $+\mathrm{V}$ " souffrir, faire mal " $3 \mathrm{p}$. sing. prés. $=$ "il (ou elle) me fait souffrir ». $3 / \mathrm{N}$ "tête " fém. sing. nomin.

" J'ai mal à la tête, moi ".

4. Verës ${ }^{1} i$-dhemb ${ }^{2}$ veshi ${ }^{3}$

$1 / \mathrm{N}$ anthroponyme " Véra " fém. sing. dat./génit. 2/ obj.2 1p. sing. + V

" faire mal » 3p. sing. prés. $3 / \mathrm{N}$ « oreille » fém. sing. nomin.

"L'oreille lui fait mal, à Véra (= "Véra a mal à l'oreille ").

5. më-vjen keq

il me vient mal

"j'ai une impression négative, mauvaise ",

c'est-à-dire: më-vjen mirë = «il me vient bien » c.à-d. «j'apprécie ».

Les expressions grecques correspondantes sont formellement identiques, aussi me contenterai-je de donner le SV dans l'ordre, à la 1p. sing. :

\begin{tabular}{|l|}
\hline mu.'pjastike o-le'mos \\
\hline «j'ai le cou bloqué » \\
\hline il me s'est bloqué le cou \\
\hline mu.po'nai to- ke'fali \\
\hline «j'ai mal à la tête » \\
\hline elle me fait mal la tête \\
\hline
\end{tabular}

61 Assez souvent, le verbe est une forme moyenne: / mu.'erxete / = "ça me vient », / mu.'fenete / = «il me paraît, j'ai l'impressions », etc. 
Exemples du grec contemporain (dhimotiki)

$\begin{array}{lll}1 & 2 & 3\end{array}$

1. tu.to.'ipes a'fto tu-pe'zju?

$1 / \mathrm{obj}_{2}$ 3p. neut. $+\mathrm{obj}_{\cdot 1}$ 3p. neut. sing. $+\mathrm{V}$ « dire $» 2 \mathrm{p}$. sing. aor. $=\ll$ tu le lui as dit». $2 /$ déict. anaph. neut. sing. $3 /$ art. $+\mathrm{N}$ « enfant» neut. sing. génit.

" tu l'as dit au gosse, ça ? "

1a. to.'ipes a'fto sto-pe'di?

Ici, le SN articulé est introduit par le relateur /s-/ et la traduction en français peut être la même que celle de l'énoncé précédent. Mais on doit supprimer l'accord objectal avec l'indice datif. Ce détail correspond au phénomène d'emphase. Maintenant, il est clair que ce procédé peut servir à lever d'éventuelles ambiguïtés, voire à éviter les formes de génitif «difficiles» pour les locuteurs contemporains, mais cela ne modifie pas fondamentalement la différence de sens entre les deux formules.

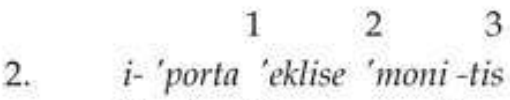

$1 /$ art. $+\mathrm{N}$ «porte fém. sing. nomin. $2 / \mathrm{V}$ «(se) fermer » 3p. sing. aor.

$3 / \mathrm{N}$ «seul » fém. sing. nomin. + poss. $3 \mathrm{p}$. fém. sing. = « d'elle même, par elle-même».

«la porte s'est fermée toute seule»

3. to-pe'di 'eklise tin-'porta

$1 /$ art. $+\mathrm{N}$ « enfant» neut. sing. $2 / \mathrm{V}$ « fermer $» 3$ p. sing. aor. $3 /$ art. + $\mathrm{N}$ «porte " fém. sing. accc.

«l'enfant a fermé la porte»

4. to-pe'di tin.'eklise tin- 'porta

«l'enfant, il l'a fermée la porte ".

5. tin-'porta tin.'eklise to- pe'ô

"la porte, l'enfant l'a fermée».

Nous avons ici une forme de thématisation de l'objet avec accord obligatoire, à la différence de l'énoncé suivant qui correspond à une focalisation du SNo :

6. tin-'porta 'eklise to- pe'öi

"c'est la porte que l'enfant a fermée ".

64 Si l'on réinsère le SNs dans le segment thématique, l'accord objectal est obligatoire, ce qui correspond à une thématisation double, du SNo et du SNs :

7. tin-'porta to- pe'əri tin.'eklise

litt. " la porte, l'enfant il l'a fermée ". 
En fait, ces derniers illustrent d'une part le fonctionnement casuel, et d'autre part le « poids » de la transitivité du SV lorsque l'arrangement énonciatif est en jeu. La parenté formelle de cet ensemble de schèmes avec ce que l'on observe en albanais est évidente.

Pour les dialectes grecs nordiques ou le pontique, on peut dire que la dativité a investi la totalité du domaine objectal. Dans ces variétés, la classe des N neutres n'a plus de marques actancielles. Les ambiguïtés éventuelles quant au rôle Bénéficiaire sont levées, au besoin, par l'utilisation du relateur locatif-allatif /s-/ qui transforme le SN considéré en circonstant :

$$
\begin{aligned}
& 1 \quad 2 \quad 3 \\
& \text { ta- ipa } a^{\prime} f t a \text { sto- } p e^{\prime} \partial t \\
& \text { 1/ obj. 3p. neut. sing. }+\mathrm{V} \text { « dire » 2p. sing. aor. = « je les(neut.) ai dit ». } \\
& \text { 2/ déict. anaph. neut. plur. } 3 / \text { loc.art. }+\mathrm{N} \text { « enfant " neut. sing. } \\
& \text { « je l'ai dit à l'enfant (tout) ça ». }
\end{aligned}
$$

Cette situation rappelle évidemment ce que l'on observe dans l'ensemble bulgaromacédonien, qui a perdu ses cas, ainsi que dans le roman oriental (roumain, aroumain, méglénite).

Dans le bref examen que nous venons d'effectuer, nous avons vu que le grec aussi bien que l'albanais disposaient de moyens formels qui permettent de marquer un actant, unique, dans le cas des verbes moyens intransitifs, ou situé en deuxième position morpho-syntaxique, dans les constructions inverses, du type «à moi l'oreille fait mal ». Nous devons préciser que ces formules sont extrêmement fréquentes et qu'elles correspondent, du point de vue sémantique, au fait que l'actant ainsi marqué est le siège d'une expérience physique, cognitive ou qu'il reflète une relation possessive, tout à fait comparable à ce que l'on observe dans certaines langues romanes comme le français, par exemple : « la maison est à moi ».

Quant à l'économie formelle que manifestent les systèmes casuels, qu'il s'agisse du génitif/ datif ou de ce qu'il convient d'appeler l'accusatif/datif, on remarque que les fusions attestées sont équivalentes du point de vue fonctionnel. C'est-à-dire que le fait de marquer un COD (complément d'objet direct) comme un COI (complément d'objet indirect) est équivalent à marquer un COI comme un COD (cf gréco-pontique, pluriel albanais, etc .) En fait, l'accord objectal qui reflète la tendance à l'équilibre formel du noyau prédicatif, soit, dans nos langues, un SV, montre que les marques ne se confondent pas et que l'indice datif a la prééminence sur les autres. On peut dire qu'en cas d'ambiguiité éventuelle, l'accusatif par défaut ou COD sera l'élément qui, pour des raisons sémantiques, ne peut être interprété comme un Bénéficiaire/Expérient.

Jusqu'ici, les similarités observées se manifestent dans un contexte de parenté génétique étroite. L'intérêt typologique des faits sera plus net si l'on observe une problématique analogue dans une autre famille linguistique.

\section{Du côté du « datif kartvèle »}

71 L'expression est de N.J. Marr, qui a souvent fait allusion à l'importance de ce cas. La famille karvèle comprend le géorgien, le svane, le mingrélien et le laze. Ces langues sont habituellement rangées au nombre des «langues ergatives». Normalement, le SN 
correspondant à l'actant unique d'un verbe considéré comme intransitif est au cas dit absolutif (nominatif dans la terminologie de Marr), de même que le SN correspondant au deuxième actant d'une construction transitive. Dans ce dernier cas, l'actant correspondant à l'Agent porte une marque dite ergative :-man en géorgien ancien, -m, ma en géorgien moderne, -k en laze, -ko, -ke en mingrélien, etc. Le SV transitif est maximaliste, au sens où il porte toujours la marque d'au moins deux actants. Le système verbal est foncièrement aspecto-modal (aspect perfectif/imperfectif, futur, optatif, atestimonial ou médiatif).

L'ergativité, dont la marque provient d'un déictique postposé fonctionnant comme thématisateur, est clivée de diverse façon selon les sous-groupes. En laze, par exemple, il s'agit d'une question de répartition diasystémique : les dialectes orientaux (Xopa, Arhavi, Vice) ont l'ergatif, le dialecte occidental de Ardešen ne connaît pas cette marque (dans cet ensemble, le cas absolutif s'oppose simplement au datif kartvèle en -s, et aux cas spatiaux, allatif, ablatif, etc.). En géorgien, le clivage est intrasystémique et il dépend de l'aspect. Prenons, par exemple, l'énoncé type du géorgien ancien :

\begin{tabular}{|l|l|l|l|}
\hline 1. Imperfectif & k'aci & c'ers & c'erilsa \\
\hline & absolutif/nomin. & V prés. & datif \\
\hline & «l'homme & écrit & la lettre « \\
\hline 2. Perfectif : & k'acman & dac'era & c'erili \\
\hline & ergatif & V aor. & absolut./nomin. \\
\hline & «l'homme & a écrit & la lettre « \\
\hline 3. Perfect./Parfait (+médiatif, narratif) : & k'acsa & uc'eries & c'erili \\
\hline & datif & V pft. 3p.sing. & datif \\
\hline & «à l'homme & a été écrite & la lettre « \\
\hline
\end{tabular}

73 On voit que le datif, appelé par N. Marr datif-accusatif, peut représenter un SN objet, Patient et/ou Bénéficiaire, mais aussi un Agent - au parfait, qui est en réalité une construction inverse. Cette formule est extrêmement fréquente. Quelques exemples permettront de résumer la forme du SV :

\begin{tabular}{|l|l|l|l|}
\hline 1. Verbe « actif » courir : & v.rbi & x.rbi & rbis \\
\hline & 1 p.sing. & 2 p. sing. & 3 p. sing \\
\hline & « je cours » & « tu cours » & « il court » \\
\hline 2. Verbe « inverse » haïr : & m.dzul.s & $=$ & « je le hais / nous le haïssons » \\
\hline
\end{tabular}




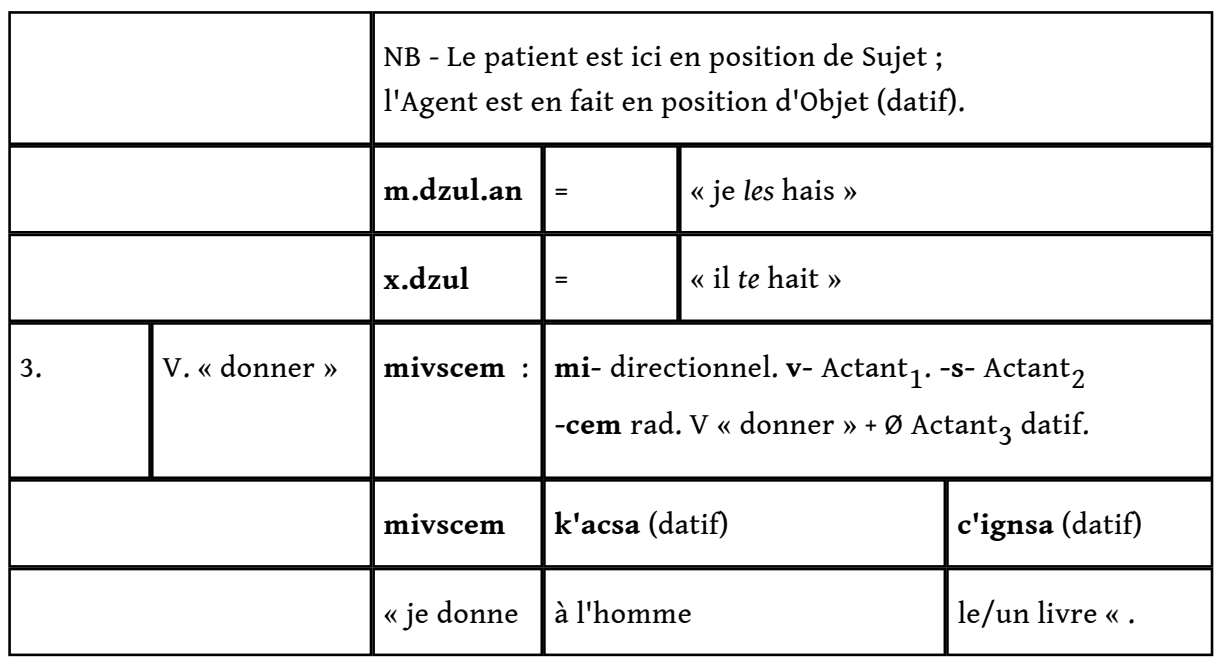

\section{Pour ouvrir une piste de recherche : La relation dative est-elle primaire?}

Les rapprochements suggérés dans notre parcours rapide ont manifesté la prégnance du datif, tant du point de vue formel que du point de vue sémantique. En considérant les données grecques et albanaises, on constate que le datif/génitif, aussi bien que l'accusatif/ datif,constitue le pivot formel d'un système où l'objet peut être marqué dans les mêmes termes qu'une relation Bénéficiaire/Expérient. Ce simple fait conforte la nécessité d'abandonner l'ordre des actants (premier, deuxième et, éventuellement, troisième actant) qui reposait sur une hiérarchie implicite mettant en tête le Sujet, puis le Complément d'Objet direct, enfin le "Complément indirect », ce dernier étant en quelque sorte marginalisé à l'instar des compléments circonstanciels. Il est clair que cette conception traditionnelle encourageait la tendance à réifier la notion de structure accusative, opposée radicalement à toutes les autres.

Si, dans l'opération consistant à mettre en relation une marque actancielle avec un rôle sémantique, la notion de Sujet a encore une utilité, le poids égal de l'Agent et du Bénéficiaire/Expérient devrait être admis. Dans la typologie contemporaine, le renversement de la hiérarchie évoquée a été réalisé, par exemple, chez T. Givón. En rejoignant cette ligne, féconde pour la question de l'accord objectal et des constructions dites « inverses", on pourrait opérer avec la notion de dativité, ce qui permettrait peutêtre de rendre plus précise la «transitivité ", dans la mesure où les liens de celle-ci avec la matrice sémantique sont assez fréquemment problématiques.

En grec et en albanais, les formes moyennes des noyaux de SV introduisent, en termes sémantiques, l'intéressement de l'élément situé comme «sujet ». C'est ce que J. Humbert avait déjà formulé, à sa façon, en opposant l'objectif (p.ex., un verbe transitif) au subjectif (un verbe moyen, transitif ou intransitif), dans un système qui connaissait par ailleurs un vrai passif ainsi que des formules inverses (dont l'argument central était au datif) au moins aussi nombreuses qu'en albanais. À cette étape de la réflexion, on doit admettre que la grille traditionnelle des « rôles » - Agent, Patient, Bénéficiaire/Expérient - risque fort d'être réductrice du strict point de vue sémantico-référentiel. Le recours à la notion de degré d'affectation (affectedness) du Patient et/ou de l'Agent, n'est pas toujours motivé. 
Dans cette perspective, le débat typologique bute sur la façon dont les divers descripteurs théorisent le lien entre la morpho-syntaxe et la composante sémantique.

77 Les études cognitives exercent actuellement une pression sur nos pratiques afin que soient dépassées les apories dont on s'accommodait sans trop de mal, avec parfois des allusions voilées aux "visions du monde» dont les langues traceraient les contours comme des sortes d'arpenteurs de l'univers sensible. L'espace alimente de ses métaphores diaprées un métalangage que des tentatives récentes s'efforcent de dépasser, non sans mal. Au sein de l'ensemble évoqué, le travail de R. Langacker (1991) propose des pistes de recherche qui pourraient être fécondes; il se montre encore influencé profondément par une hiérarchie fonctionnelle où la transitivité fonde le modèle de toute relation bi-actancielle, mais il a l'audace de renverser la hiérarchie des marques (p. 246 sq.). Dans son optique, il serait «normal » que l'éléments le plus bas sur l'échelle des rôles soit souvent le plus marqué ; Agent et Patient constituent les prototypes du Sujet et de l'Objet direct, et le Datif est ancré dans l'archétype de l'Expérient. Il a une intuition suffisamment importante pour devoir être citée (p. 253) :

«Because cases represent separate predications, and correlate only partially and contingently with grammatical relations, it is hardly surprising that even subjects are sometimes marked with role-based cases, notably dative. »

Ce cadre permettrait sans aucun doute d'expliquer nombre des faits que nous avons brièvement examinés, en reconnaissant les effets d'un "tendance naturelle », pour reprendre les termes de Langacker. Mais cela nous laisse sans voix devant nos syntagmes verbaux lourds, qui amalgament les indices en une sorte de maximum anaphorique, avec des traces casuelles évidentes. La tendance à constituer un noyau rhématique complexe, observée aussi bien en albanais qu'en grec (pour ne pas parler des langues kartvèles, exemplaires à cet égard), va de pair avec des clivages formels qui, s'ils ont la même origine, renvoient à deux domaines différents. Dans le système nominal, les asymétries relevées correspondent essentiellement à la dialectique Quantification/Qualification. Le système verbal, quant à lui, exhibe des différences dans les marques énonciatives qui constituent les racines du réseau anaphorique.

On pourrait avancer l'idée que le domaine de l'objectalité comme expérience permet à l'énonciateur de se construire à partir d'un clivage fondamental dont le « datif » constitue une trace formelle.

\section{BIBLIOGRAPHIE}

ASsatiani, I., MALHerbe, M. (1997) Parlons géorgien, Paris , L'Harmattan, 284 p.

DEMIRAJ, S. (1985) Gramatikë historike e gjuhës shqipe, Sht. botue. « 8 nëntori », Tiranë, 1166 p.

DIXON, R.M.W. (1994)Ergativity, Cambridge University Press, 271 p.

DRETTAS, G. (1994) "Structure ergative ou structure accusative : le cas du gréco-pontique dans une perspective typologique et aréale", Actances 8, Paris, p. 203-224. 
(1997) “Aspects pontiques”, Paris, Arp, LXVIII, + 789 p.

(1987) "Problèmes de la linguistique balkanique", Bulletin de la Société de Linguistique de Paris,

LXXXII-1, Klincksieck, Paris, p. 257-281.

GIVóN, T. (ed.) (1994) Voice and Inversion, Amsterdam/Philadelphia, John Benjamins Publishing

Company.

GIVóN, T. (1995) Functionalism and Grammar, Amsterdam/Philadelphia, John Benjamins Publishing

Company.

GJINI Niko H., Fjalor Shqip-Greqisht, Sht. botue. e librit shkollor, Tiranë, 1971.

Gramatika e Gjuhës shqipe. v. I : Morfologjia, Tiranë, 1995.

GUENTCHEVA, Z. (1994) Thématisation de l'objet en bulgare, Berne, Peter Lang.

HARRIS, A. (1985) Diachronic Syntax, the Kartvelian Case, New York, Academic Press (Syntax and

Semantics 18). XXIII + $463 \mathrm{p}$.

HEWITT, B.G. (1995) Georgian. A Structural Reference Grammar, Amsterdam/Philadelphia, John

Benjamins Publishing Company.

HORROCKS, G. (1997) Greek. A history of the Language and its Speakers, London-New York, Longman

Linguistics Library.

HUMBERT, J. (1982) Syntaxe grecque, Paris, Klincksieck.

Kemmer, S. (1993) The Middle Voice, Amsterdam/Philadelphia, John Benjamins Publishing Company.

LANGACKER, R.W. (1991) Concept, Image and Symbol. The Cognitive Basis of Grammar, Berlin / New York, Mouton de Gruyter.

LAZARD, G. (48/1995) « Le géorgien : actance duale (« active ») ou ergative ? Typologie des verbes

anti-impersonnels », Sprachtypologie und Universalienforschung (STUF) 48, p. 275-293.

MARR, N. et BRIÈRE, M. (1931) La langue géorgienne, Paris, Firmin Didot.

LÜDERS, U. (1992/1) “Ergativity and variability actant marking in Pazar-Laz”, LINCOM 3, p. 24-35.

PËRNASKA, R. (1996) La Syntaxe de l'énoncé en albanais contemporain, Thèse de doctorat ès-Lettres,

Université de Paris VII, 671 p. (ex. dactyl.).

SANDFELD, K. (1930) La Linguistique balkanique,. Problèmes et résultats, Paris, H. Champion.

SASSE, H.J. (1991) Arvanitika, Wiesbaden, Otto Harrassowitz.

VOGT, H. (1988) Linguistique caucasienne et arménienne, Oslo, Norwegian University Press.

\section{NOTES}

1. Les toponymes, les anthroponymes et les noms des langues sont transcrits ou translittérés selon le système le plus fréquent appliqué à chaque langue. Ainsi le graphème $x$ par exemple, qui sert à translittérer le signe cyrillique correspondant, peut correspondre au $h$ de l'albanais ou du turc.

Pour les exemples albanais, l'orthographe officielle est utilisée. Mais les exemples toskesarvanites de Grèce empruntés à la description de H.J. Sasse, qui sont écrits en API, n'ont pas été modifiés. Les exemples grecs sont écrits en API. Le géorgien est écrit selon le système utilisé actuellement par les caucasologues français. 


\section{AUTEUR}

GEORGES DRETTAS

C.N.R.S. - PARIS 